\title{
VI.
}

\section{Die Behandlung des Freiheitsproblems bei John Locke.}

\author{
Von \\ Dr. A. Messer, Giessen.
}

I. Einleitung.

1. "Unsere Aufgabe in dieser Welt", bemerkt Locke am Anfange seines Essays $\left.{ }^{1}\right)(I, 1, \S 6)$, „ist nicht alles zu wissen, sondern die Dinge zu kennen, die auf unser Leben von massgebendem Einfluss sind". „Man kann mit Grund schliessen“, heisst es an einer anderen Stelle (IV, $12, \S 11$ ), „dass unsere Aufgabe in den Untersuchungen und in der Art. von Kenntnissen enthalten ist, die unsern natürlichen Fähigkeiten am meisten entsprechen, und die unsere grössten Angelegenheiten betreffen; d. h. unsern Zustand in der Ewigkeit. Deshalb dürfte die Moral die wahre Wissenschaft und Aufgabe der Menschheit im Allgemeinen sein." Zutreffend erklärt Monroe Curtis ${ }^{2}$ ): "The entire writings of Locke bear a practical and ethical impress". Die durchaus praktische Richtung, die schon Bacons Denkweise kennzeichnet und die bei

1) Ich citiere den englischen Text nach der Ausgabe: The works of John Locke, in nine Volumes. The twelfth edition. London 1824. Bei der Uebersetzung schliesse ich mich meist an die in Kirchmanns „philosophischer Bibliothek" gegebene an.

2) An outline of Locke's ethical philosophy. Leipz. Diss. 1890. S. 23. - Vgl. auch Conduct of Underst. §23. Works II p. 360. 
dem Nachlassen dos Interesses an rein theoretischen, metaphysischtheologischen Controversen im 17. und 18. Jahrhundert immer mächtiger sich geltend macht, beherrscht auch ihn. Der Moralphilosophie und der Theologie, die ihm ja die unentbehrliche Grundlage für die Moral zu bieten schien, war stets sein Interesse zugewandt, wenn er auch nicht dazu kam, seine ethischen Ansichten systematisch zusammenzufassen. V. Hertling ${ }^{3}$ ) weist daraufhin, dass jene Erörterungen im Freundeskreise, die, nach Lockes Angabe im „Brief an den Leser“, den Anstoss za-den im Essay niedergelegten Untersuchungen gaben, sich „um die Principien der Moral und der geoffenbarten Religion drehten"; er knüpft daran die ansprechende Vermutung ${ }^{4}$ ), dass auch die Tendenz des Essays in dieser Richtung zu suchen sei: dadurch, dass ein „die Gesammtheit der Wirklichkeit umspannendes systematisches Wissen " als von vornherein aussichtslos nachgewiesen wird, wird auch die Gefahr beseitigt, dass von einer solchen Naturphilosophie aus sich Consequenzen ergeben, die Moral und Religion erschüttern könnten.

2. Unter den ethischen Fragen scheint aber Locke das Problem der Willensfreiheit in ganz besonderer Weise zum Gegenstande seines Nachdenkens gemacht zu haben. Davon zeugt die ausführliche Erörterung, die er über diesen Gegenstand in das 21. Kapitel des 2. Buches seines Essays eingeschoben hat. Sie allein hat auch, wie er ausdrücklich hervorhebt ${ }^{5}$ ), bei der 2. Auflage seiner Schrift wirkliche Abänderungen erfahren, und auch von der 6. Auflage hören wir ${ }^{6}$ ), dass ihre meisten Zusätze auf eben dieses Kapitel sich beziehen. Weil also Locke gerade dieser Materie eine ernste und dauernde Aufmerksamkeit zugewandt hat, dürfte es sich empfehlen, seinen Erörterungen darüber einmal genau nachzugehen.

3. Locke hat nun aber ganz bestimmte ethisch-religiöse Grundanschauungen, die auch durch die erkenntniss-theoretischen Unter-

3) John Locke und die Schule von Cambridge. Freiburg i. B. 1892. S. 245 .

4) A. a. 0. S. $244-274$.

5) Brief an den Leser. Uebers. S. $24 \mathrm{ff}$. und Essay II, 21, § 72.

6) Br. a. d. Les. Uebers. S. 28. 
suchungen, die er in seinem Essay niederlegt, durchaus nicht erschüttert worden sind. „Das Dasein Gottes und die Existenz einer geistigen Welt neben und über der materiellen wird ebensowenig in Frage gezogen wie der Bestand eines objektiven, allverbindenden Sittengesetzes; vielmehr bilden die hierauf gerichteten Ueberzengungen die festen Schranken, innerhalb deren die Untersuchung

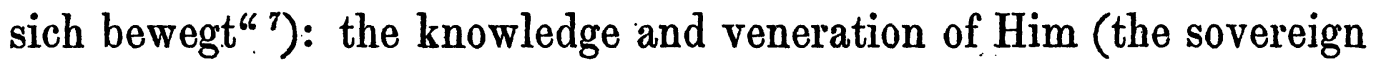
disposer of all things) being the chief end of all our thoughts, and the proper business of all understandings (II, 7, §6). Dass Locke infolgedessen auch an das Problem der Willensfreiheit mit ganz bestimmten Voraussetzungen herantrat, die sein Ergebniss beeinflussen mussten, ist an sich wahrscheinlich und wird von ihm ausdrücklich bestätigt, wenn er sagt: God having revealed that there shall be a day of judgement, $J$ think that foundation enough to conclude men are free enough to be made answerable for their actions, and to receive according to what they have done $^{8}$ ). Es ist deshalb unerlässlich auf eben diese ethisch-religiösen Grundanschauungen Lockes zunächst einzugehen.

4. Ferner gilt auch von Lockes Darstellungsart die Bemerkung Falckenbergs über diejenige Descartes': „Mit seiner schlichten, naiven, mehr weltmännischen als gelehrten Denk- und Redeweise vertrug sich eine subtile Feststellung und strenge Festhaltung sicherer Termini überhaupt nicht" ${ }^{\text {9 }}$ ). Deshalb soll auch seine vielfach schwankende Terminologie, soweit sie für unsere Frage in Betracht kommt, einer besonderen Erörterung unterzogen werden. Nach Constatierung der hieraus sich ergebenden Schwierigkeiten kämen wir zu unserer eigentlichen Aufgabe, nämlich seine Untersuchung über das Freiheitsproblem in ihrem logischen Fortschreiten genau zu verfolgen und sodann die sich durchziehenden Grundgedanken herauszulösen. hinweist.

7) v. Hertling a. a. 0 . S. 265 , der auch auf die oben citierte Stelle

8) Monroe Curtis a. a. 0. S. 87 aus einem Briefe Lockes an Molyneux v. 20. Jan. 1693. Works IV. 278.

9) Geschichte der neueren Philosophie. 2. Aufl. Leipzig. 1892. S. 84. 


\section{Religiöse und othische Grundansichten Lockes.}

1. Es ist neuerdings mit Recht darauf hingewiesen worden ${ }^{10}$ ), dass in Lockes Denken zwei verschiedene Tendenzen neben einander hergehen, deren Antagonismus bisweilen deutlich auf die Oberfläche tritt. Die eine dieser Richtungen, die rationalistische, findet ihre Stütze in seinem festen christlichen Glaubén, die andere, die empiristische, ist getragen von der gleichgerichteten geistigen Strömung jener Zeit, die schon in Bacons Schriften zum wirkungsvollon Ausdruck gekommen war. Jene zeigt sich mehr in seinem gewissermassen von den Vätern ererbten Gedankenbesitz, dièse charakterisiert mehr sein eigenes philosophisches Erwerben.

Indem wir die Grundzüge seiner religiös-sittlichen Weltanschauung überblicken, wollen wir nicht künstlich Geschlossenheit und Einheitlichkeit in dieselbe hinein interpretieren, sondern es nicht unbeachtet lassen, dass an verschiedenen Stellen seiner Schriften bald mehr die eine, bald mehr die andere Richtung seines Denkens hervortritt, sodass manche Aeússerungen in der That nicht wohl im Einklang zu bringen sind.

2. Die unerschütterliche Ueberzeugung von der Existenz eines persönlichen, unendlich vollkommenen Gottes bildet einen Grundpfeiler in Lockes Gedankenbau. Nicht minder stand ihm fest, dass alle Menschen durch das Licht der Vernunft die Existenz Gottes erkennen können, da die Schöpfung deutliches Zeugnis von ihm ablege. Diese Anschauungen teilte er mit der ganzen mittelalterlichen Theologie und Philosophie, die ja auch damals noch, in ihren Grundzügen unverändert, die Schulen beherrschte ${ }^{11}$ ).

10) Vgl. die bei v. Hertling a. a. O. S. 3 genannten Schriften, auch H. selbst widmet diesem Nachweise das erste Kapitel seines Buches. Übrigens hat schon Kant darauf hingewiesen, „der berühmte Locke" leite die reinen Verstandesbegriffe aus der Erfahrung ab, verfahre aber „doch so inconsequent, dass er damit Versuche zu Erkenntnissen wage, die weit über alle Erfahrungsgrenze hinausgingen". (Kritik d. r. V. S. 111 Ausg. v. Kehrbach.)

11) v. Hertling macht mit Recht hierauf aufmerksam. (A. a. 0. S. 58.) Er giebt auch (S. 48) mehrere Stellen an, wo L. die Erkenntnis Gottes als naheliegend für den Menschen erklärt. Zu vergleichen ist hier auch die Berner Dissertation von W. Küppers, J. Locke und die Scholastik. Berlin 1895. 
Dass Locke die relativ leichte Erkennbarkeit Gottes wiederholt mit dem grössten Nachdruck betont, hat aber noch einen besonderen Grund.

Religion und Ethik ist für ihn, wie für die christlichen Denker vor ihm, auf das engste verknüpft. Ohne Gottes Gesetzgebung und die von ihm ausgehende. Sanktion des Gesetzes kann er sich keine Sittlichkeit denken ${ }^{12}$ ). Andererseits kann es ihm aber nicht zweifelhaft sein, dass das Gericht Gottes über die Menschen gerecht sein werde; dass „jedem vergolten werde nach seinen Werken“, und dass „niemand weiter als wegen seiner eigenen Sünden gestraft

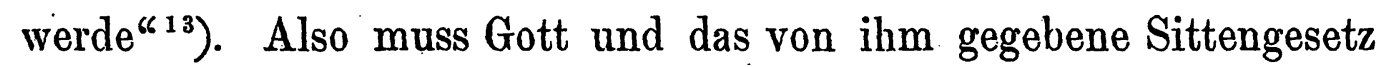
auch ohne Offenbarung den Menschen erkennbar sein ${ }^{14}$ ), da sie sonst von Gott nicht zur Rechenschaft gezogen werden könnten.

Die nie wankende Festigkeit seines eigenen Gottesglaubens liess ihm das, was ihm schon vor jedem Beweis feststand, als so klar beweisbar erscheinen, dass er zu der Ansicht gelangt: Gott nicht finden, heisse ihn nicht finden wollen. Nur wer unter keinem Gesetz leben wolle, ziehe die Existenz eines obersten Herrschers und eines allverbindenden Gesetzes in Zweifel ${ }^{15}$ ).

Es lässt sich nun aber deutlich darthun, wie von den Voraussetzungen Lockes aus, soweit sie in der empiristischen Richtung liegen, sich allenfalls noch die Bildung der Gottesidee erklären, nicht aber die objektive Realität derselben erweisen lässt ${ }^{16}$ ), so dass schon hier die Zwiespältigkeit seines Denkens bemerkbar ist.

3. Das Verhältnis Gottes zu den Menschen wird ebenfalls der traditionellen Auffassung entsprechend gedacht. Das Ziel des Menschen soll sein: „der Ruhm und die Ehre Gottes und seine

12) Vgl. über diesen Punkt Essay I, 3, § 12. II, 28, §6.

13) The reasonableness of Christianity (Works VI. p. 8, 9).

14) Essay. III, 9, §23: „And we ought to magnify his goodness that he hath spread before all the world such legible charakters of his works and providence, and given all mankind so sufficient a light of reason, that they to whom this written word never came, could not (when ever they set themselves to search) either doubt of the being of a God, or of the obedience due to him".

15) Lord King, life of J. Locke (London 1830) I, 166 bei v. Hertling a. a. 0. S. 49. A.

16) v. Hertling, a. a. 0. S. 60. 
eigone Gläckseligkeit" ${ }^{17}$ ). (The honour and veneration of the Creator, and the hapiness of mankind.)

In Jockes Denken tritt nun hauptsächlich der lotzte Gesichtspunkt (die Glückseligkeit als Bestimmung der Menschheit) beherrschend hervor: es ist durchaus eudaimonistisch. Die Ethik ist ihm die Wissenschaft, welche die Regeln und Normen des menschlichen Handelns aufsucht, die zur Glückseligkeit führen, und die Mittel zu ihrer Verwirklichung an die Hand gibt ${ }^{18}$ ).

Bei seiner eudaimonistischen Grundanschauung wird er doch nie an der Güte Gottes irre, weil sein Denken zugleich durchaus optimistisch ist ${ }^{19}$ ). Er ist überzeugt: das zeitliche Leben ist trotz seiner vielen Mängel besser als gar keines; es ist eine besondere Gnade Gottes, wofür ihm die Menschen zu Dank verpflichtet sind, wenn er ihnen auch nur ein zeitliches und sterbliches Leben verleiht ${ }^{20}$ ).

Man wird anderseits sagen dürfen, dass sein fester Glaube an die Güte Gottes seinen Optimismus auch da aufrecht erhält, wo ihm die Beobachtung und Erfahrung (also die empiristische Richtung in ihm) Momente bietet, die an sich geeignet wären, ihn zu zerstören; solche werden bisweilen sogar unter der Wirkung einer unwillkürlich eintretenden Interpretation, in ihr gerades Gegenteil verkehrt. So wird die Thatsache, dass Gott den Dingen die Kraft beigelegt hat, nicht nur Lust-, sondern auch Schmerzempfindungen zu erregen, als ein Beweis für Gottes Güte aufgefasst ${ }^{21}$ ).

17) Conduct of the understanding $\S 23$.

18) Essay IV, 21, §3: „ethics, which is the seeking out those rules and measures of human actions, which lead to happiness, and the means to practise them.

19) Wenn dies nicht der Fall ist, liegt es nahe, von eudaimonistischen Grundanschauungen aus, von dem Glauben an die Güte Gottes und damit an die Existenz eines persönlichen Gottes überhaupt abzulenken. Einen Beleg dafür bietet in der späteren Entwicklung der englischen Ethik auf der gleichen eudaimonistischen Basis James Mill. „Er fand es unmöglich zu glauben, dass eine Welt so voll Uebel das Werk eines Urhebers sei, der mit der Allmacht eine unendliche Güte und Gerechtigkeit verbinde." (John Stuart Mills Selbstbiographie übers. v. C. Kolb. S. 32.)

$\left.{ }^{20}\right)$ Reas. of Chr. (Works VI p. 7 sq.).

21) Essay. II, 7, § 3 sq. 
Es liessen sich ferner eine ganze Rẹ̣he von Anschauungen des „Empiristen“ Locke zusammenstellen, die den Anschein erwecken könnten, als deuteten sie auf eine pessimistische Weltauffassung ${ }^{22}$ ). Aber in Wirklichkeit neigt Locke niemals einer solchen zu. Sein Glaube an einen gütigen Gott und seine optimistische Denkweise, in Wechselwirkung sich stützend und stärkend, lassen negative Instanzen nicht zur Geltung kommen.

4. Auch in Lockes Ansichten über die oberste sittliche Norm und über das Sittengesetz machen sich zwei nicht vereinbare Auffassungen geltend. Es sind im wesentlichen dieselben, die auch in der scholastischen Philosophie hervorgetreten waren.

Für die Nominalisten war das Sittengesetz etwas willkürlich von Gott Gebotenes. "Gott fordert Gehorsam gegen das Sittengebot, nicht weil es gut ist, sondern weil es sein Gebot ist $\left.{ }^{23}\right)$. "Deshalb kann auch nicht die Vernunft, sondern nur die Offenbarung Erkenntnisquelle dafür sein.

Die Realisten sahen in der vernünftigen Menschennatur die

22) Es ist z. B. ein von ihm stark betontes Ergebnis seines empiristischen Denkens, dass gerade das „Unbehagen“ es ist, was den Willen in Bewegung setzt (Essay II, $21 \S 35$ ); dabei macht sich fast fortwährend so vielerlei Unbehagen geltend, dass der Mensch sich selten ganz behaglich füblt (II, 21 $\S 45)$; nun genügt aber auch ein kleines Unbehagen, um all unser Glück zu zerstören (II, 21, $\S 36, \S 64$ ), auch wirkt die Lust nicht so stark auf uns wie der Schmerz (II, 20, § 14), so ist es wohl begreiflich, dass in diesem Leben nur wenigen Glück beschieden ist, und auch diesen nur ein sehr mässiges (II, 21, § 44). Um zu beweisen, dass demungeachtet es besser sei zu leben als nicht zu leben, genügt ihm der Hinweis auf den Selbsterhaltungstrieb der Menschen (Reas. of Chr. Works VI p. 8): einer weniger optimistischen Denkweise könnte dieser gerade als eine fatale Fessel erscheinen, die den Menschen an sein trübseliges Dasein kettet. - Fernerhin bemerkt er, dass nur wenige Menschen dazu kommen, ihre natürlichen Anlagen zu entwickeln und zu der des Menschen wärdigen Erkenntnis gelangen, da „der träge und unbedachtsame Teil der Menschen die grösste Zahl ausmache" (Essay I, 4, § 15). Auch in Bezug auf die sittliche Kraft des Menschen ist er wenig zuversichtlich: er glaubt aus der Erfahrung entnehmen zu müssen, dass grosse körperliche Schmerzen (auch heftige Leidenschaften II, 21, §12) eine $\mathrm{z}$ wingende Nacht auf den Willen ausüben (II, 21, §57); er knüpft daran unbefangen die Bemerkung, wir hätten demnach allen Grund zu beten: „Führe uns nicht in Versuchung".

23) Wundt, Ethik, S. 266. 
unmittelbare Norm des Sittlichen, die mittelbare und höchste aber in der göttlichen Wesenheit; denn die Idee des Menschen ist eine von den unendlich vielen möglichen Abbildern göttlicher Vollkommenheit, die von Ewigkeit her im Geiste Gottes ruhen. Insoforn ist sie ihren wesentlichen Zügen nach wandellos; insofern ist es aber auch ein für allemal gegeben, dass ein bestimmtes Verhalten eben das der vernünftigen Menschennatur entsprechende (und damit das sittliche) ist. Dieses lässt sich durch vernünftige Betrachtung aus der Menschennatur ableiten, das Sittengesetz (lex naturae) ist also durch das licht der Vernunft erkennbar.

Die nominalistische Auffassung kommt bei Locke zur Geltung, wo or sich in seinen empiristischen Gedankengängen bewegt.

Wie das Erkennen so soll auch das Handeln des Menschen aus möglichst einfachen Principien abgeleitet werden. Die Fịhigkeit Lust- und Schmerzempfindungen zu haben ist alles, was ihm nach der praktischen Seite hin angeboren ist. Gott hat die Dinge mit solchen Kräften ausgestattet, dass sie neben den Vorstellungen auch derartige Empfindungen wecken; deshalb sind diese auch fast allen unseren Vorstellungen beigemischt, und sie sind es, die den Menschen überhaupt erst zum Wollen und Handeln anregen ${ }^{24}$ ).

Der Fähigkeit der Dinge, Empfindungen zu erregen, muss im Menschen eine bestimmte Empfänglichkeit entsprechen, damit eine Empfindung überhaupt zustande komme. Hier entgeht seiner Beobachtung die Thatsache nicht, dass diese Empfänglichkeit individuell verschieden ist. „Der Wohlgeschmack hängt nicht von dem Gegenstande ab, sondern davon, ob er dem einzelnen Gaumen entspricht; hier besteht aber eine grosse Verschiedenheit und deshalb liegt das grösste Glïck in dem Besitze der Dinge, welche die grösste Lust gewähren und in der Entfernung von allem, was Schmerz und Störung verursacht, und dies sind für die einzelnen sehr verschiedene Dinge." Er leitet daraus die Folgerung ab: "Setzt man daher seine Hoffnung nur auf dieses Leben, so ist es weder befremdlich noch unvernünftig (not strange nor unreasonable), wenn man das Glück in der Vermeidung von allem, was hier un-

24) Essay II, 7. § 1-5. 
angenehm ist und in Verfolgung von allem, was hier ergötzt, sucht $\left.{ }^{25}\right)$."

Hier wird also Locke durch den empiristisch-nominalistischen Zug in seinem Denken zum Moralpositivismus geführt, wie ihn die nominalistischen Scholastiker aus der Schule eines Duns Scotus, Occam und Gerson vertreten hatten; und wie ihn in der damaligen Zeit auch Descartes verfócht. „Der einzige Grund alles Existirenden, aller Wahrheit, aller Güte und alles Rechts ist allein der absolut unbeschränkte; schlechthin indifferente Wille Gottes, dessen Entscheidungen wir nicht anders, als zufällig nennen können $\left.{ }^{26}\right)$.

Wäre das Jenseits nicht - so lautet also hier die Ansicht Lockes - mit seinen für bestimmte Arten der Lebensführung von Gott (willkürlich) festgesetzten Belohnungen und Strafen, so wäre es vernünftig (also auch sittlich: wenn man dann hiervon überhaupt reden könnte), wenn jeder seine individuellsten Gelüste zu befriedigen suchte; eine für alle verbindliche Norm des Handelns gäbe es schlechterdings nicht.

Das Sittengesetz erscheint als etwas durchaus dem Menschen an sich Fremdes, Heteronomes, von aussen an ihn Herantretendes, das der Grundlage in der Menschennatur entbehrt; denn die einzigen angeborenen praktischen Principien: das Streben nach Lust und die Flucht vor Unlust würden, "wenn man ihnen volle Freiheit gäbe, die Menschen zur Vernichtung aller Moralität führen ${ }^{27}$ )“.

Es giebt ausser dem Gesetz durchaus keine Norm des Sittlichen. Es wird nicht etwa deshalb etwas für den Menschen zum

25) II, 21, §55.

26) Fr. Jodl, Geschichte der Ethik in der neueren Philosophie I (Stuttg. 1882) S. $259 \mathrm{f}$.

${ }^{27}$ ) Essay. I, 3, § 13. - Dass eine (allerdings nicht sehr tief eindringende) Empirie zu solchen Anschauungen leiten kann, ist einleuchtend. Zu allen Zeiten wird sich der Beobachtung die Thatsache dargeboten haben, dass sehr viele Menschen dem Sittengesetz als einem ihnen innerlich Fremden, Heteronomen gegenüber zeitlebens verharren, dem sie nur infolge der imperativen Notive des äusseren und inneren Zwangs gehorchen. Gerade auf diese Punkte hatte auch Locke seine Aufmerksamkeit gerichtet, wie daraus hervorgeht, dass er neben dem göttlichen und dem bürgerlichen Gesetz „das Gesetz.der guten Meinung und des Rufes" (the law of opinion or reputation) statuiert II, $28, \S 10$. 
Gesetz orhoben, woil es schon an und für sich dem Menschen als vornünftigen Wesen angemessen (und darum ngut") wäre, sondern durch das Gesot» wird das „Gute“ und „Böse“ (im ethischen Sinne) überhaupt erst geschaffen. „Gut und Uebel ist nur Lust und Schmerz oder das, was sie uns verschafit. Das moralische Gut und Uebel (moral good and evil) ist die Uebereinstimmung oder Nicht-Uebereinstimmung unserer freien Handlungen mit einem Gesetz, wobei das Gut und Uebel durch den Willen und die Macht des Gesetzgebers über uns gebracht wird ${ }^{28}$ )."

Dabei macht sich auch seine durchaus eudaimonistische Denkweise geltend. Das sittlich Gute ist nicht etwas von dem Guten im Sinne des Lustbringenden Verschiedenes; es bildet hier nur insofern eine gewisse Unterart desselben, als es nicht direkt auf unsere Empfindung einwirkt, sondern erst mittelbar durch die Belohnung, die der Gesetzgeber damit verknüpft hat und die uns eben Lust erregt. Das Analoge gilt für das Böse.

Ohne diese Sanktion d. h. ohne die mit ihnen verbundene Belohnung und Bestrafung wären Gesetze gänzlich nutzlos. Das Willkürliche dieser Verknüpfung wird dabei noch ausdrücklich hervorgehoben durch die Bemerkung, dass das Uebel oder die Belohnung sich nicht schon von selbst (also durch den natürlichen Zusammenhang) aus der Handlung ergeben dürfte, da sonst die Vorschriften überflüssig wären ${ }^{29}$ ). -

Dem allem gegenüber treten aber auch hier ganz andersartige Anschauungen hervor. V. Hertling hat schon darauf hingewiesen, wie selbst mitten in den durchaus empiristischen Gedankengängen des ersten Buches der Essays die Existenz eines, durch die Vernunft erkennbaren, natürlichen Sittengesetzes (der lex naturalis der Scholastik) von Locke ausdrücklich anerkannt wird ${ }^{30}$ ). So werden auch nebeneinander, als augenscheinlich in innigster Beziehung

$\left.{ }^{28}\right)$ Essay. II, 28, \$5. - Schon die Scholastik hatte dagegen geschieden zwischen dem bonum delectabile und utile und dem bonum honestum (naturale und morale).

29) II, 28, §6.

30) A. a. 0. S. 228 mit Bezug auf Ess. I, 3, § 13. - Ueberhaupt verweise ich zur näheren Begründung der Bemerkungen über sittliche Norm und Sittengesetz auf die Darlegungen v. Hertlings. S. $220 \mathrm{ff}$. 
stehend genannt: das ewige Gesetz und die Natur der Dinge (the eternal law and nature of things II, $21, \S 56$ ). "Gott", so bemerkt er gelegentlich II, 7, $\S 4$, „hat mit der Einwirkung gewisser Dinge auf unseren Körper Schmerz verbunden, um vor dem von ihnen drohenden Schaden zu warnen." Er erkennt damit unwillkürlich an, dass Gott diese Verbindung angeordnet hat entsprechend der Natur dieser Dinge, die eben an sich schon unserem Körper schädlich waren, auch abgesehen von dem durch sie erregten Schmerz. Er redet ferner von den "natürlichen Vorschriften über Recht und Unrecht" (natural measures of right and wrong $I, 3, \S 11$ ), und erkennt an, dass Tugend und Laster durch ihre eigne Natur recht und unrecht seien. (II, 28, 10.)

Damit harmoniert es, dass er es für notwendig erklärt, die Wahrheit und Vernünftigkeit (the truth and reasonableness) der Moralsätze nachzuweisen (I, 3, § 4). Er nennt die Pflichten zwischen den Eltern und Kindern solche, die sich am unmittelbarsten aus der Vernunft ergeben (I, 3, § 12). Wie wäre dies möglich, wenn sie lediglich auf willkürlicher Anordnung Gottes beruhten. Er erklärt geradezu: „Die Vorstellung eines höchsten Wesens von unendlicher Macht, Güte und Weisheit, dessen Werk wir sind, und von dem wir abhängen, und die Vorstellung unserer selbst, als vernünftiger Wesen, - Vorstellungen, die ganz klar sind - bieten bei gehöriger Betrachtung und Untersuchung solche Grundlagen für unsere Pflichten und für die Regeln des Handelns, dass die Moral dadurch zu den Wissenschaften, die des. Beweises fähig sind, erhoben werden kann. Gewiss würden auch hier, von selbstverständlichen Sätzen aus, vermittelst der Folgerungen so sicher wie in der Mathematik die Grenzen von Recht und Unrecht von denen dargelegt werden können, die ihnen dieselbe Unbefangenheit und Aufmerksamkeit wie anderen Wissenschaften zuwenden". (IV, 3, § 18.) $\left.)^{31}\right)$

31) Hier sei auch eine Bemerkung über Lockes Ansicht von der mathematischen Beweisbarkeit des Sittlichen gestattet. Für die Gleichsetzung des wissenschaftlichen Charakters der Mathematik und der Moral lassen sich im Sinne Lockes folgende Gründe geltend machen.

1) Die mathematischen wie die moralischen Vorstellungen sind mixed 
Solcho Auschauungen lassen sich natiirlich aus den oben gekenuzeichneten empiristischen und nominalistischen Voraussetzungen

modes; die Seole bildet sie, ohne dabei zu fragen, ob sie wirklich 80 in der Natur bestehen oder nicht (II, 22, § $2 ; 30, \S 4)$. Sie sind deshalb sämtlich ontsprechend, ineil sie keine $A b$ bilder wirklich bestehender Dinge sein sollen" (II, 31, §3), sondern „Urbilder“. „Sie sind nur für solche Zustănde bestimmt, die, wenn sie bestehen, dann auch genau mit ihnen übereinstimmen" (II, 34, § 14).

2) Die mathematischen wie die moralischen $\mathrm{Satze}$ entstehen durch $\mathrm{Be}$ ziehung der betr. Vorstellungen aufeinander: sie fallen also unter die zweite der 4 von Locke IV, 1, § 3 unterschiedenen Wissensarten. „Die Vorstellung eines höchsten Wesens von unendlicher Macht, Güte und Weisheit, dessen Werk wir sind, und von dem wir abhängen, und die Vorstellung unserer selbst, als vernünftiger Wesen, welche Vorstellungen so klar sind, bieten bei gehöriger Betrachtung solche Grundlagen für unsere Pflichten und für die Regeln des Handelns, dass die Moral dadurch zu den Wissenschaften, die des Beweises fähig sind, erhoben werden kann." (IV, 3, § 18.) Die mathematische Beweisbarkeit erstreckt sich also auch „auf die sittlichen Grundurteile“; nicht nur auf Ableitung der „Folgesätze“ daraus und auf die Beurteilung, welche Handlungen mit denselben übereinstimmen, welche nicht (wie Jodl annimmt Gesch. d. Ethik in d. n. Phil. I Stuttg. 1882. S. 154). Noch ein anderes Beispiel führt Locke (a. a. 0.) an: „Wo es kein Eigentum giebt, da giebt es auch kein Unrecht; dies ist ein Satz so sicher wie irgend ein Lehrsatz des Euklid; denn die Vorstellung des Eigentums ist das Recht auf eine Sache, und die Vorstellung, die Unrecht genannt wird, ist der Einbruch in dieses Recht oder seine Verletzung. Bei solcher Feststellung der Vorstellungen und der ihnen gegebenen Namen kann die Wahrheit dieses Satzes ebenso sicher erkannt werden; als dass die 3 Winkel des. Dreiecks 2 Rechten gleich sind." (Auf diese Stelle nimmt Hume in seinem Enquiry (Essays, London 1784 vol. II p. 173; Kirchmanns Uebers. S. 151) ausdrücklich Bezug. Er erklärt, der mathematische Satz lasse sich " nicht ohne eine Reihe von Gründen und Betrachtungen einsehen", er liefere deshalb auch eine wirkliche Erkenntnis; „aber zum Beweis, dass wo kein Eigentum ist, es auch keine Ungerechtigkeit giebt, genügt die Definition der Worte und die Erklärung, dass Ungerechtigkeit in der Verletzung des Eigentums bestehe“. Das sei aber keine neue Erkenntnis. Er erklärt deshalb „die Grösse und die Zahl für die einzigen Gegenstände der Vernunftwissenschaft und der strengen Beweise" - the only proper objects of knowledge and demonstration.)

3) Die mathematischen und moralischen Sätze gewähren ein wirkliches $\mathrm{W}$ issen; denn wie die Vorstellungen aus denen sie bestehen, wollen sie die Wirklichkeit nicht abbilden, sondern vorbildlich für sie sein. Die Sätze über den Kreis und das Viereck gelten, „mag ein Kreis oder Viereck in der Welt bestehen oder nicht“. „Des Cicero Pflichtenlehre bleibt nicht weniger wahr, wenn auch niemand in der Welt diese Regeln beobachtet. “ (IV; $4, \S 8$.$) .$ 
nicht ableiten, ja sie widerstreiten ihnen geradezu : sie entstammen eben der rationalistischen Seite seines Denkens.

Die hiermit anerkannte. Bedeutung der Vernunft für die Erkenntnis des Sittlichen wird uns aber in Lockes Erörterung über die Freiheit wieder begegnen.

5. Aus dem Gesagten wird aber jedenfalls soviel ersichtlich sein, dass er dem Problem der Willensfreiheit selbst gar nicht voraussetzungslos gegenüberstehen konnte. Die Grundzüge seiner religiösen und ethischen.Weltauffassung haben sich uns durchaus als die traditionell-christlichen ergeben: er konnte also auch ein für das christliche Denken so wichtiges Problem wie das der Willensfreiheit nicht gänzlich "losgelöst von diesen Grundanschauungen betrachten.

Diese Annahme findet in Lockes eigenen Aeusserungen ihre volle Bestätigung. Als Beispiel eines zutreffenden Syllogismus benutzt er: die Kette von Vorstellungen: „die Menschen werden gestraft werden - Gott straft - eine gerechte Strafe - der Bestrafte ist schuldig - er hätte anders handeln können - Freiheit - Selbstbestimmung“ (IV, 17, §4). Man wird -vermuten dürfen, dass diese Vorstellungsverbindung seinem Denken sehr geläufig war, weil er einen solchen Gebrauch von ihr machte.

Die Freiheit muss also in dem Sinne von dem Menschen bejaht werden, dass ihm dadurch Selbstbestimmung und Verantwortlichkeit für sein. Handeln zugeschrieben wird: diesen festen Punkt bot ihm sein Christentum ${ }^{32}$ ); ebendaher stammt die Ueberzeugung, dass ein von Gott gegebenes Sittengesetz die Norm für das menschliche Handeln abzugeben habe. In der Anschauung aber, dass Lust- und Schmerzempfindung die einzigen ursprünglichen Triebfedern des Handelns seien, traf seine empiristische Denkrichtung mit seiner eudaimonistischen Gefühlsweise zusammen.

32) Daran wurde er auch durch den Umstand nicht irre, dass er die Vereinbarkeit der Willensfreiheit mit der Allmacht und Allwissenheit Gottes nicht erfassen konnte. $I$ cannot make freedom in man consistent with omnipotence and omniscience in God, though I am as fully persuaded of both as of Any truths. I most firmly assent to": Brief an Molyneux vom 20. Jan. 1693. Works IV 278 bei Monroe Curtis a. a. 0.87. 


\section{Bemerkungen über die Terminologie Lockes.}

1. Eine grundlegende. Unterscheidung ist für Locke geradeso wic für Descartes, diese: es giebt 2 Arten von Thätigkeit: Denken und Bewegen. (Two sorts of action, whereof we have any idea, viz. thinking and motion.) ${ }^{33}$ ).

Als die diese Thätigkeiten veranlassenden Kräfte werden genannit: Auffassungs- bezw. Denkkraft und Bewegungskraft oder Wille: perceptivity or power of perception or thinking II, $21, \S 73$, (wofür denn auch in gleicher Bedeutung understanding gebraucht wird II, $21, \S 18$ ) und motivity or power. of mowing II, 21, $\S 73$, was weiterhin gleichgesetzt wird mit will II, $23, \S 18,{ }^{34}$ ) so dass also der Wille zusammenfiele mit der vis motrix der scholastischen Philosophie.

Es liegt hierbei aber wohl nur eine ungenaue Ausdrucksweise Lockes vor, auf die er lediglich da verfiel, wo er die beiden Gebiete körperlichen und geistigen Geschehens, Bewegung und Denken, gerade ins Auge fasste.

$\mathrm{Da}$, wo er den Willen selbst zu definieren unternimmt, ist èr weit entfernt, sein Wirken auf das erste dieser Gebjete einzuschränken. So bezeichnet er II, $21, \S 15$ den Willen als eine Kraft der Seele, ihr Denken zur Hervorbringung, Fortführung oder Hemmung einer.Handlung so weit zu bestimmen, als diese Handlung von ihr abhängt. (The power of the mind to determine its thought, to the producing, continuing, or stopping any action, as far as it depends on us.) Uebereinstimmend damit sagt er II, 21, § 28: "Man muss festhalten; dass das Verlangen oder Wollen (volition or willing) eine That der Seele (act of the mind) ist, insofern sie ihr Denken auf die Hervorbringung einer Handlung (action)

33) Essay. II, 21, § 4. Wiederholt wird diese Unterscheidung II, § 8 und in der zusammenfassenden Betrachtung $21, \S 71$.

34) The ideas we have belonging, and peculiar to spirit, are thinking and will, or a power of putting body into motion by thougt. Aehnlich II, 21, 16, wo in Bezug auf den Willen sich die Bezeichnung findet: the power that is in a man to produce or forbeare producing motion in parts of his body, by choice or preference. 
richtet und dabei ihre Macht zu deren Hervorbringung ausübt. Es giebt aber, nach seiner oben dargelegten Auffassung zwei Arten von Handlung: Denken und Bewegen. Also wird er dem Willen nicht nur die Veranlassung von Bewegungen zuschreiben müssen, sondern auch die von Denkakten.

Dies wird nun auch von ihm ausdrücklich ausgesprochen: „So viel dürfte wenigstens gewiss sein, dass man in sich eine Kraft zum Beginnen oder Anhalten, zum Fortfahren oder Beenden jener verschiedenen Thätigkeiten der Seele und Bewegungen des Körpers bemerkt, welche lediglich durch ein Denken oder Vorziehen der Seele gleichsam das Vollziehen oder Nicht-Vollziehen von solch einer einzelnen Handlung anordnet oder befiehlt. Diese Kraft der Seele, vermöge deren sie die Betrachtung einer Vorstellung (consideration of any idea) oder deren Nichtbetrachtung anordnet, oder die Bewegung der Ruhe eines Gliedes oder das Umgekehrte in jedem einzelnen Falle vorzieht, ist das, was man Wille (will) nennt. Die wirkliche Ausübung dieser Kraft durch Bewirkung oder Unterlassung einer einzelnen Handlung ist das, was man Wollen (volition or willing) nennt" (II, 21, §5).

Der Wille ist also die Grundkraft der Seele, die Kraft, „welche die wirkenden Vermögen des Menschen (the operative faculties of a man II, 21, §29) zur Bewegung oder Ruhe bestimmt, soweit sie von dieser Bestimmung abhängig sind“.

Man darf auch, im Anschluss an Lockes Betrachtungs- und Ausdrucksweise sagen: Der Wille ist dasjenige, durch dessen Beobachtung sich unserer Selbstwahrnehmung (reflection) die Vorstellung der "thätigen Kraft" (active power) als Eigenschaft der Seele ergiebt, denn die Aufmerksamkeit auf die Vorgänge in unserem Innern gewährt uns „die Vorstellung einer Kraft zum Beginnen einer Thätigkeit: Bewegen oder Denken" ${ }^{35}$ ), d. i. einer thätigen.

35) II, 21, § 4, wo der Ausdruck active power nachher erklärt wird durch: power to begin any action, either motion or thought. Kirchmann übersetzt hier nicht zutreffend: „Kraft, die ein Thun oder Bewegen oder Denken anfängt. (S. 249.) - Die „Sensation“ ergiebt nach Locke nur eine sehr dunkle Vorstellung von sthätiger Kraft", weil die Wahrnehmung an den Körpern: nur die Uebertragung, nicht die Hervorbringung einer Bewegung zeigt. 
Kraft, und diese ist eben, nach der oben gegebenen Definition, der Wille.

2. Es hat sich also ergeben, dass nach Lockes Auffassung die Bethätigung des Willens eben sowohl in der Anordnung geistiger Akte wie in derjenigen körperlicher Bewegungen besteht. Erinnern wir uns nun der von ihm vollzogenen Scheidung aller Thätigkeit in Denken und Bewegen und fragen wir, zu welcher Art er die Willensbethätigung selbst zu rechnen habe, so kann kein $\mathrm{Zweifel}$ darüber sein, dass er sie der ersteren zuweisen müsse. Wirklich finden sich auch Stellen, an denen der Ausdruck „Denken“ (thinking, thought) zur Bezeichnung geistiger Thätigkeit überhaupt gebraucht wird. So lesen wir II, 21, § 17: „Man fasst den Willen und den Verstand als Vermögen, welche die Handlungen des Wählens und Verstehens vollführen sollen, obgleich sie doch nur ver-. schiedene Arten des Denkens sind." (We make the will and understanding to be faculties, by which the actions of choosing and perceiving are produced, which are but several modes of thinking.) Ebenso werden Willensakte (volition, willing, preference) als Denkakte (thought) ${ }^{36}$ ) und einmal, in ganz cartesianischer Weise, das Wollen als ein "Modus des Denkens" bezeichnet ${ }^{37}$ ).

Dem gegenüber macht sich allerdings auch das Bedürfnis geltend, auf dem geistigen Gebiete selbst eine Scheidung vorzunehmen, die Thätigkeiten des Erkennens von denen des Wollens zu sondern und beide auf besondere Vermögen zurückzuführen. So wird denn im Anschluss an die oben citierte ausführliche Definition des Willens (II, 21, §5), von diesem scharf unterschieden: die Kraft des Auffassens oder der Verstand (the power of perception is that which we call the understanding). Dabei wird nun der Ausdruck „Denken“ auch zur Bezeichnung der Verstandesthätigkeit

(II, 21, § 4.) An einer anderen Stelle sagt er geradezu, sie gewährten nur die Vorstellung des „Vermögens bewegt zu werden“ (power of being moved), also die Vorstellung der „leidenden Kraft" (passive power). (II, 21, § 73.)

36) Vgl. II, 21, §5; § 11, § 21, § 28.

37) II, 19, § 2: Especially since I shall have occasion hereafter to treat more at large of reasoning, judging, volition, and knowledge, which are some of the most considerable operations of the mind, and modes of thinking. 
allein gebraucht. So kommt es, dass die Termini think und thought, die an den oben angegebenen Stellen zum Ausdruck geistiger Thätigkeiten überhaupt und demnach auch des Wollens dienten, gelegentlich auf den Verstand allein bezogen und geradezu gegensätzlich zu Ausdrücken wie volition angewendet werden. So werden geschieden (II, 21, § 18): power of thinking und power of choosing; (II, 21, $\S 8$ und $\S 19$ ): thought und volition, will; (II, 21, $\S 9$ ): think und volition or preference. 
